\title{
La Zona de Desarrollo Próximo como tarea de construcción
}

\author{
MARÍA DEL MAR GONZÁLEZ y JESÚS PALACIOS \\ Universidad de Sevilla
}

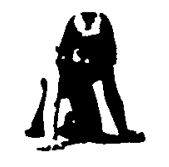

Resumen

\begin{abstract}
Desde una perspectiva vygotskiana, las interacciones adulto-niño son observadas en torno a una tarea de construcción, analizándose las diferencias existentes entre unos adultos y otros en la forma en que definen la situación y la negocian en la interacción con niños de entre 21 y 24 meses de edad. Los análisis efectuados con la muestra total de 133 padres (hombres y mujeres) y sus respectivos hijos, muestran que los indices de estimulación de las madres (no de los padres) y los indices de desarrollo de sus bijos, guardan entre sí una correlación interesante, aunque baja. El sexo de los padres, y no su nivel de estudios, se relaciona con el nivel de estimulación aportada. Los análisis secuenciales realizados sobre una submuestra de madres estimulantes y otra de madres poco estimulantes, evidencian que hay distintas formas de interactuar con el niño en su Zona de Desarrollo Próximo, y que las diferentes relaciones interpersonales conducen a distintos niveles de competencia por parte de los niños.
\end{abstract}

Palabras clave: Interacción, Andamiaje, Zona de desarrollo próximo, Estilos educativos.

\section{The Zone of Proximal Development as a construction task}

\section{Abstract}

From a Vygotskyan viewpoint, adult-infant interactions are observed around a construction task, the differences existing between adults in terms of the way in which they define the situation and negotiate it throughout the interaction with an infant 21-24 months-ald being analysed. The analyses carried out with a total sample of 133 subjects (busbands and wifes) and their respective sons and daughters, show that mothers (but not fathers') stimulation scores and their infants' development scores, have an interesting (but low) correlation. Parents' sex, and not their degree of education, is related to the level of stimulation shown up in the interaction. Sequential analyses carried out with two subsamples of mothers (high and low in stimulation), show that there are different ways of interacting with the infant in bis Zone of Proximal Development, and that those differences are associated with different levels of children's competence.

Keywords: Interaction, Scaffolding, Zone of proximal development, Educational styles.

Agradecimientos: Varias son las personas que en distintos momentos y para distintas fases de la investigación de que aqui se informa han aportado una colaboración que para nosotros ha sido de enorme valor. M." Carmen Moreno en primer lugar e Isabel García, Rafael Martínez y José Antonio Pérez Gil constituyen la nónima de las personas a las que debemos expresar nuestro más sincero agradecimiento por su colaboración eficaz y directa en este trabajo. Estamos también muy agradecidos a $R$. Bakeman, por habernos facilitado con toda amabilidad su programa de análisis secuencial, y a I. Vila, que nos puso en contacto con el programa SALT para análisis del lenguaje por ordenador y nos lo facilitó.

Dirección de los autores: Psicología Evolutiva y de la Educación. Universidad de Sevilla. Avda. San Francisco Javier, s/n. 41005 Sevilla. 
En su reciente exposición de la evolución y el estado actual de la Psicología Evolutiva en la Unión Soviética, Valsiner (1988) dedica un capítulo especial a L. S. Vygotsky. Al referirse a él dice que la aceptación por parte de Vygotsky de la filosofía marxista, no fue la de un seguidor ardiente; en vez de limitarse a hacerse eco de los grandes axiomas del marxismo, Vygotsky fue "un activo creador de psicología marxista» (Valsiner, 1988, p. 125). Respecto a quienes simpatizan con el enfoque vygotskyano tal vez se pueda hacer una distinción similar entre quienes se limitan a ser «ardientes seguidores» de Vygotsky (repitiendo aquí y allá sus principios fundamentales, estén mejor o peor entendidos y hagan más o menos al caso), y quienes son «activos creadores» de psicología vygotskyana. Si la obra y el enfoque de Vygotsky se han hecho tan populares y tan influyentes en los últimos años, tal vez haya sido en gran parte porque hemos tenido la suerte de contar con excelentes y activos creadores de psicología vygotskyana que no sólo nos han acercado al enfoque y los postulados de Vygotsky, sino que han sido capaces de desarrollarlos, de mostrarlos en acción, de llevarlos más allá. Los nombres de Bruner y Wertsch deben ser citados en lugar preferente, y tras ellos los de otros muchos investigadores que han aportado su contribución a una mejor comprensión de cómo ocurre el proceso sociogenético de construcción del desarrollo. El presente trabajo trata de aportar una modesta contribución a esta tradición investigadora concentrándose en las interacciones educativas adulto-niño y tratando de mostrar algunos aspectos diferenciales que existen en la forma en que los padres crean desarrollo en la interacción con sus hijos.

En su excelente presentación del pensamiento y la obra de Vygotsky, Wertsch (1985) ha mostrado los diferentes dominios genéticos existentes en la teoría vygotskyana: la filogénesis, la historia sociocultural, la ontogénesis y la microgénesis. Muchas de las investigaciones de inspiración vygotskyana se han desarrollado en el dominio de la microgénesis, que es una especie de «estudio longitudinal a corto plazo" (Wertsch, 1985, p. 71 de la trad. cast.) en el que se analiza de qué manera se forma una competencia o un proceso psicológico determinado en un intervalo de tiempo razonablemente breve y en el curso de un proceso de interacción. Tales investigaciones han tenido éxito a la hora de demostrar la virtualidad de los dos grandes principios que inspiran toda la psicología de Vygotsky: el principio del origen social de los procesos psicológicos superiores y el principio de que no todos los procesos de enseñanza/aprendizaje generan desarrollo, sino sólo aquéllos que se sitúan en la zona de desarrollo próximo (ZDP) del niño.

El primero de estos principios afirma que los procesos psicológicos superiores son el resultado intrapersonal de procesos interpersonales en los que uno de los interactuantes (en nuestro caso, el padre o la madre) es capaz de construir en el otro procesos que no existían y que la interacción ha sido capaz de generar. El proceso eficaz de enseñanza/aprendizaje es aquél en el que quien tiene mayor control de la interacción educativa logra transferir al interior de quien aprende contenidos o destrezas de que éste carecía. Uno de los elementos distintivos de la aproximación vygotskyana es que esta transferencia se realiza a través y en el curso de procesos de interacción, tal como se ha mostrado en diversas investigaciones (por ejem- 
plo, Rogoff y Gardner, 1984; Rogoff, Malkin y Gilbride, 1984; Wertsch, 1985; Wertsch y Hickman, 1987).

El segundo de los principios indicados se refiere al hecho de que no toda interacción produce desarrollo o avance en quien es guiado. Para ser eficaces, las interacciones educativas deben situarse en la ZDP del niño, definida como la capacidad para el desarrollo guiado que existe en el niño en cualquier momento de su desarrollo. Si respecto a una capacidad o destreza determinada, el niño no ha accedido aún a un cierto nivel de competencia, pero puede acceder a ella con ayuda y guía, entonces decimos, con Vygotsky, que tales ayudas han sido eficaces porque se han situado en la ZDP del niño. Cuatro aspectos nos parecen cruciales en relación con el concepto de ZDP y a ellos nos vamos a referir a continuación.

El primero de ellos se refiere al hecho de que la ZDP no es algo preexistente en el niño que el adulto se limite a hacer manifiesto a través de la interacción, pero tampoco es la mera trasposición al interior del niño de todo lo que se pone a su disposición en el exterior. La ZDP es creada en el niño por el proceso de enseñanzalaprendizaje (Vygotsky, 1978, p. 138 de la trad. cast.) ${ }^{1}$. Sobre la base del desarrollo que el niño ya tiene, el adulto es capaz de llevarle más allá si cumple con ciertas reglas que nos permiten seguir caracterizando la noción de ZDP.

El segundo aspecto al que se refiere la ZDP ( $y$ una de las reglas a que acabamos de aludir), se relaciona con la noción de "definición de la situación de interacción". Este concepto es particularmente importante y lleva en su interior dos nociones relacionadas: la de intersubjetividad y la de asimetría (Wertsch, 1984). Toda situación de interacción implica un cierto nivel de intersubjetividad, de percepción y acción conjunta. Cuando la interacción se da entre adulto-niño y en ella el primero trata de enseñar algo al segundo, hay una evidente asimetría entre el que sabe y el que aún no sabe, siendo entonces de la mayor importancia la forma en que el adulto se plantea la tarea y se la plantea al niño, es decir, el contexto de enseñan$\mathrm{za} /$ aprendizaje que el adulto crea en torno a la tarea (Wertsch, Minick y Arns, 1984). Así, por ejemplo, si pedimos a la madre que construya una pirámide con el niño sirviéndose de las piezas de distintos tamaños y colores que les proporcionamos, algunas madres pueden plantear la tarea (es decir, definir la situación) como si la meta fundamental fuera construir la pirámide (sin importar mucho quién lo hace y cómo lo hace), mientras que otras pueden hacerlo como si la meta fundamental fuera que el niño adquiriera en esa tarea todas las competencias que su nivel de desarrollo le permita (sin importar tanto si al final la pirámide está o no construida). Algunas madres actúan como si la tarea fuera una sucesión de episodios sin relación entre sí, mientras que otras enfocan la tarea más bien como el aprendizaje paulatino de las reglas que rigen su realización.

- Cuando el adulto propone al niño una determinada definición de la situación, le está proponiendo, en el mejor de los casos, una especie de «borrador» de contrato (Rommetveit, 1985), que es suficientemente flexible como para cambiarse si es necesario. La tarea puede planteársele al niño completamente al alcance de sus posibilidades actuales (por ejemplo, el adulto la realiza y el niño sólo tiene que mirar; o el adulto deja al niño que realice una parte de la misma que no le requiere ningún esfuerzo), completamente alejada de sus posibilidades actuales (por ejemplo, pidiéndole al 
niño que la haga solo, o sin tener en cuenta si realmente entiende o no las instrucciones que se le dan), o como una actividad en la que se van a aprovechar las destrezas que el niño ya tenga, pero se va a tratar de potenciar otras nuevas que están al alcance de sus posibilidades de desarrollo y aprendizaje (por ejemplo, el niño ya sabe coger y colocar piezas, y eso se le pide que lo haga desde el principio, pero aún no sabe seguir ninguna regla en la realización de la tarea, y se va a tratar de que aprenda alguna de ellas).

Una vez que el adulto crea el contexto, es decir, define la situación de interacción, otro importante proceso se pone en marcha, proceso que constituye el tercer rasgo de la ZDP y otra de las reglas de la interacción si se quiere que ésta dé lugar a desarrollo: la negociación de la definición aportada por el adulto. Tras poner en marcha el proceso, el adulto presta atención a las claves que el niño le proporciona en la interacción; estas claves le informan de que la definición propuesta hace la tarea demasiado sencilla para el niño (la definición dada por el adulto se sitúa en el nivel de desarrollo actual del niño), o demasiado complicada (cuando se sitúa más allá de la competencia posible a partir de la competencia ya alcanzada). Si el adulto es sensible a estas claves, modificará su definición de la situación de manera que resulte retadora y estimulante para el niño, permitiéndole acceder a nuevos niveles de competencia. Como ha señalado McLane (1987), la ZDP debe ser negociada entre el niño y el adulto que le guía. El proceso de interacción constructiva adulto-niño es en gran parte un permanente proceso de negociación en el que el adulto explora los límites de la ZDP y trata de ensancharlos todo lo posible.

Por fortuna, sabemos bastante sobre cómo funciona ese proceso de negociación. Wood (1980; Wood, Bruner y Ross, 1976; Wood y Middleton, 1975) lo ha descrito de manera particularmente clara con la metáfora del andamiaje, que ha ilustrado también con una tarea de construcción (DeLoache y DeMendoza, 1987, han hecho una ilustración semejante en una tarea de lectura conjunta de libros). El proceso de andamiaje tiene dos rasgos fundamentales: según el primero de ellos, el adulto sitúa la definición de la tarea algo por encima de las capacidades actuales del niño, de tal manera que, como acabamos de señalar, la situación sea retadora. De acuerdo con el segundo rasgo, la intervención del adulto es inversamente proporcional al nivel de competencia mostrado por el niño (regla de contingencia); apoyándole de manera más directiva cuando el niño carece de competencia, y retirando luego progresivamente el apoyo a medida que la va adquiriendo (proceso de desandamiaje). El éxito del proceso de enseñanza/aprendizaje depende en gran medida de la capacidad de quien guía el proceso para subir y bajar oportunamente el nivel de exigencia, para adecuar las demandas y los apoyos al nivel de competencia que el niño ya tiene y a las capacidades de que dispone para acceder a nuevos niveles, manteniendo a la vez el interés del niño por la tarea y promoviendo en él un sentimiento de confianza en sus posibilidades de ejecución. El éxito del proceso depende, en una palabra, de la habilidad del adulto para negociar al alza la ZDP del niño.

Finalmente, el cuarto rasgo del concepto de ZDP se relaciona con el concepto de desafio semiótico (Wertsch, 1985, p. 185), que está íntimamente conectado con los procesos que acabamos de describir. El desafío forma parte de la negociación al alza que el adulto plantea, que implica reto y es- 
fuerzo. Lo que hay que añadir a continuación es el carácter semiótico que suele adoptar ese reto, al realizarse a través de un instrumento mediador esencial: el lenguaje. Desde un momento muy temprano en el desarrollo del niño, el lenguaje es la moneda fundamental de intercambio en el proceso de interacción social que genera el desarrollo de los procesos psicológicos superiores en el niño. Pero el lenguaje a través del cual se establece la intersubjetividad puede contener niveles muy diversos de desafío. Así, por ejemplo, en la interacción en la que padre e hija están implicados en la construcción de una pirámide, el primero puede decirle a la segunda "pon ahora esta pieza", o puede preguntarle «¿cuál viene ahora?», o, a un nivel intermedio, puede decirle «ahora vamos a poner la pieza roja grande». El universo de significados que el adulto maneje en la interacción resultará ser crítico a la hora de determinar el universo de significados a que podrá acceder el niño en el plano intrapsicológico.

De estas diversas cuestiones trata la investigación de la que se informa en este artículo. En ella tratamos de poner a prueba una serie de hipótesis alrededor del proceso en el que adulto y niño interactúan. Tratamos de probar si, como suponemos, los adultos difieren unos de otros en la forma en que definen la situación para el niño. Tratamos de probar también si, como suponemos, las diferencias se dan no sólo en lo que concierne a la situación de partida, sino también a la negociación subsecuente: de acuerdo con nuestras hipótesis, habrá adultos que serán más capaces que otros de ir ajustando los niveles de exigencia y apoyo que plantean al niño a los niveles de competencia mostrados por éste, de manera que se maximice la virtualidad constructiva de la interacción. Esperamos, pues, encontrar en los adultos de nuestra muestra distintos estilos de interacción educativa, definidos por la forma en que definen y negocian a través del andamiaje la situación educativa.

Pero el análisis microgenético que aquí se hace, está hecho en parte sobre la base de que sus datos proyectan información relevante para la comprensión de la construcción ontogenética. Cuando el investigador se decide por una aproximación microgenética es en gran medida como consecuencia de una elección estratégica: se trata de estudiar en una situación concreta procesos que se supone operan (en la misma pareja de interactuantes) en muchas otras situaciones interactivas. Así, cuando encontramos un determinado estilo de interacción madre-niño alrededor de una tarea concreta, suponemos que ese estilo se relaciona con la forma habitual de interacción de esa pareja en otras muchas situaciones y tareas. Ello nos permite suponer que existirá una cierta relación entre el estilo de interacción microgenéticamente estudiado y el proceso de desarrollo cuando es analizado desde una perspectiva ontogenética, que es una de las hipótesis generales de nuestra investigación.

Otra de las hipótesis que manejamos se relaciona con el hecho de que cuando un adulto guía el desarrollo de un niño, lo hace no desde un estilo de interacción estrictamente individual, sino relacionado con determinantes de tipo cultural, histórico y social. Lo que un adulto pone en juego en su interacción educativa es un conjunto de expectativas, actitudes y destrezas que ha adquirido como parte de su proceso de desarrollo en un contexto cultural, histórico y social determinado. De hecho, cuando nos interesamos por las diferencias existentes entre distintos grupos sociales esta- 
mos adoptando un enfoque en cierto modo histórico-cultural, pues en una sociedad desigual los distintos grupos sociales presentan distintos niveles de apropiación de la cultura y acceden incluso a contenidos culturales diferentes, así como a diferentes ideologías, representaciones y scripts en relación con la educación de los hijos.

A nuestro entender, estos dos aspectos (relación microgénesis-ontogénesis, diferencias entre grupos sociales) han recibido una escasa atención de parte de los investigadores anglosajones, aparentemente más preocupados por los procesos microgenéticos por sí mismos, y menos sensibles a la problemática de las diferencias intraculturales. Por nuestra parte, prestaremos atención también a esas dos cuestiones.

\section{METODO}

\section{Sujetos}

La muestra estaba compuesta de 68 niños de uno y otro sexo y sus padres. En el momento de ser estudiados, los niños tenían una media de 22 meses y medio, variando entre $21 ; 18$ y 24;9. La distribución por sexos de los niños fue desigual: 31 niños y 37 niñas. Los padres eran de nivel educativo bajo (49 sujetos), medio (41) o alto (43). Las familias fueron reclutadas para el estudio en el Hospital Maternal de la Seguridad Social en Sevilla, en los días siguientes al nacimiento de los niños, prestándose voluntariamente para la realización de un estudio longitudinal. En dos de las familias no pudo ser estudiado el padre y en otra, la grabación de la interacción madre-hijo hubo de ser invalidada a causa de la intervención de otros hermanos. Por esas razones, el número definitivo de padres estudiados no es $136(68 \times 2)$ sino 133 .

\section{Instrumentos}

Padres e hijos fueron grabados en vídeo mientras interactuaban en torno a una tarea consistente en realizar una construcción con piezas de madera: una pirámide con 12 piezas en el caso de las madres y un abeto con 16 en el de los padres (en la Fig. 1 se representa la tarea que los niños debían realizar con sus madres). Para poder realizar tales construcciones, padres e hijos debían colocar las piezas siguiendo un cierto número de reglas, dos de las cuales eran comunes a ambas tareas: la seriación de las piezas de mayor a menor y su inserción en un pivote. Una tercera regla hacía diferentes a ambas tareas: en el caso de las madres, los niños debían colocar piezas alternativamente con un escalón hacia arriba y hacia abajo, mientras en el caso de los padres la regla se relacionaba no con la orientación, sino con la forma de la pieza: debían alternar una pieza rectangular con otra redonda.

Se eligió este tipo de tareas fundamentalmente por dos razones. En primer lugar, porque su realización estaba claramente más allá del nivel de competencia en solitario de los niños, dado que nuestro interés era analizar las estrategias de interacción desarrolladas por los padres en situaciones en las que tienen la oportunidad de contribuir a que sus hijos avancen en competencia en una determinada habilidad. En segundo lugar, porque son ta- 

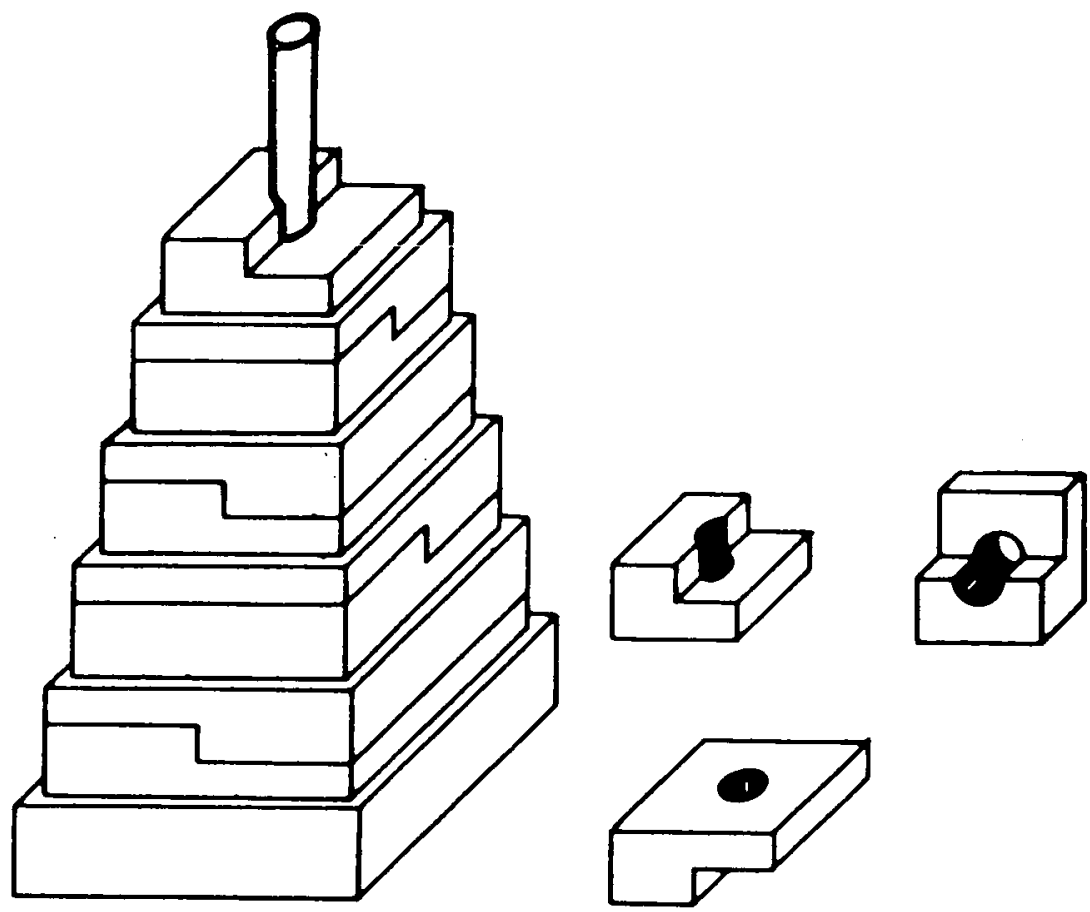

Tarea de construcción utilizada para analizar las interacciones madre-bijo.

reas que no se resuelven en una sola operación, sino cuya resolución requiere la realización repetida de un conjunto de operaciones, circunstancia que permite evaluar mejor el comportamiento de la díada.

\section{Procedimiento}

Las grabaciones se llevaron a cabo en las casas de nuestros sujetos. Antes de grabar a niños y padres, los investigadores mantenían con éstos una entrevista en la que dialogaban de modo relajado en torno a la vida cotidiana del niño, lo que permitia establecer un buen clima de relaciones entre padres y entrevistadores. A continuación se grababa a ambos padres interactuando por separado con su hijo. Mientras se grababa la interacción de uno de los padres con el niño, el otro se encontraba en una habitación distinta respondiendo a las preguntas de una entrevista, con lo que se evitaba que los padres se observaran el uno al otro durante su interacción con el niño.

La situación de interacción se planteaba a los padres explicándoles que estábamos interesados en observar el juego de sus hijos y que por tanto queríamos que hicieran con ellos lo que solieran hacer cuando encontraban un rato libre y se sentaban a jugar con ellos. Primero se grababa una interacción con los juguetes habituales del niño y a continuación les aportábamos nuestros materiales dándoles las siguientes instrucciones: "Mira, éste es un juego que suele gustar mucho a los niños. Como ves, consiste en ir inser- 
tando las piezas por este palito de mayor a menor, pero cuidando de ir poniendo una pieza con el escalón hacia arriba y otra con el escalón bacia abajo (una alargada y una redonda, en el caso de los padres varones), de modo que se acabe formando una pirámide (un abeto). Hazlo como suelas hacerlo cuando llega un juguete nuevo a casa y te sientas a jugar con el niño. Es el niño quien debe hacer la construcción, pero probablemente no pueda bacerla solo, asi que tú ayúdale si ves que lo necesita."

De las instrucciones se excluían expresamente los términos «enseñar» o "explicar", de manera que no se indujera este tipo de comportamiento en los padres, y se facilitara que cada uno interpretara a su modo la instrucción de "prestar ayuda cuando lo necesitara el niño». A continuación se les daban las piezas del juego y se grababa lo que hacían, terminando la grabación en general cuando ellos daban por concluida la interacción, tras haber realizado una o varias veces la construcción. Cuando la tarea fue realizada más de una vez por una díada, elegimos para los análisis la ejecución en la que la implicación del adulto era mayor.

La tarea de construcción, sobre la cual giran nuestros análisis en este trabajo, tiene dos subtareas: la de elección de la pieza que en cada momento corresponda, y la de su inserción en el pivote. Ello permite diferenciar, tanto en la conducta de los padres como en la de los niños, entre un conjunto de categorías relacionadas con la elección y un conjunto de categorías relacionadas con la colocación de la pieza. El sistema de categorías de observación utilizado para la codificación de los vídeos se recoge en la Tabla I. La codificación ha sido realizada de modo independiente por dos observadores, con un índice de fiabilidad aceptable ( $89 \%)$. Los desacuerdos en la codificación se resolvieron mediante la revisión de los vídeos, discusión y codificación por parte de un tercer observador.

En lo que concierne a la determinación del nivel de desarrollo de los niños, se han considerado dos índices: ley;

a) un indice global de desarrollo: la subescala mental de la escala Bay-

b) el nivel de desarrollo del lenguaje: evaluado a partir del lenguaje emitido por los niños en otra situación de interacción grabada, situación que propicia especialmente la producción lingüística: la «lectura» conjunta padres-hijo de un cuento a base de dibujos. Las transcripciones del lenguaje han sido analizadas mediante el programa SALT (Miller y Chapman, 1985) de análisis de nivel de desarrollo del lenguaje, siguiendo para el cálculo de morfemas la adaptación al castellano efectuada por Vila (1984) de las reglas de Brown (1973). La variables de lenguaje que se han considerado han sido el número total de palabras y el número de raices diferentes utilizadas por el niño. 


\section{TABLA I \\ cistema de categorias de análisis}

\section{CATEGORIAS REFERIDAS A LA CONDUCTA DE LOS PADRES}

\section{Definición de la situación}

Regla de la tarea: verbalización en la que se explica al niño la o las reglas que rigen la ejecución de la tarea, efectuando una planificación secuencial de ella («Primero se ponen los más grandes y después los más pequeños»).

Regla parcial de elección: verbalización que recurre a alguno de los términos que definen las reglas de la subtarea de elección para explicar la razón o el modo de llevar a cabo una acción relacionada con dicha subtarea ( Esa pieza no vale porque ahora toca una redonditam).

Regla parcial de colocación: verbalización que recurre a alguno de los términos que definen la subtarea de colocación para explicar la razón o el modo de llevar a cabo una acción relacionada con dicha subtarea ( EEsta pieza tienes que ponerla con el escaloncito para abajo").

\section{Niveles de intervención en la tarea}

a) Relacionados con la elección de la pieza

Instrucción verbal inespecífica: verbalización en la que se hace referencia a la siguiente pieza a elegir pero sin dar al niño las claves que le permitan conocer sus características («Y ahora, ¿cuál ponemos?").

Instrucción verbal específica: verbalización que indica al niño las características de la siguiente pieza a elegir («Busca ahora la azul grande»).

Aporte de material: el adulto señala o sencillamente da al niño la pieza que ha de poner a continuación.

b) Relacionados con la colocación de la pieza

Orientación correcta de la pieza: la madre da al niño la pieza en la orientación correcta (en el caso del padre, el problema de la orientación de la pieza no existe).

Ayuda fisica en la colocación de la pieza: el adulto comienza a colocar una pieza y deja al niño terminar la acción, o bien apoya físicamente toda la colocación de la pieza, pero siendo el niño quien claramente dirige la acción.

Toma de posesión de la actividad: el adulto lleva a cabo en solitario la colocación de la pieza, o bien ambos tocan la pieza pero el niño simplemente acompaña el movimiento director del padre.

\section{Protagonismo permitido al niño}

Demora del apoyo prestado al niño: el adulto deja un lapso temporal igual o mayor a 4 segundos entre el momento en que el niño demuestra tener dificultades en la colocación de la pieza y su intervención de ayuda.

Corrección paterna de errores: el adulto corrige la colocación incorrecta de una pieza efectuada por el niño.

Petición al niño de autocorrección: el adulto pide al niño que cambie alguna pieza que colocó de modo incorrecto ("Esta está mal puesta; quítala antes de seguir»).

\section{Soporte emocional de la actividad}

Expectativas positivas: el adulto expresa su confianza en la capacidad del niño para llevar a cabo una determinada acción («Ponla tú solo, que tú puedes»).

Aprobación: el adulto confirma al niño la corrección de su acción anterior, incluyendo o no matiz afectivo o de alabanza («Eso es, así está bien»).

Desaprabación: el adulto niega al niño la pertinencia de su acción anterior para el desarrollo de la tarea que llevan a cabo conjuntamente ("No, esa pieza no es»).

\section{CATEGORIAS REFERIDAS A LA CONDUCTA DEL NIÑO}

Elección correcta: el niño elige correctamente la pieza que ha de colocar a continuación.

Elección incorrecta: el niño elige de modo incorrecto la siguiente pieza a colocar.

Colocación correcta: el niño coloca de modo acertado la pieza.

Colocación errónea: el niño introduce de modo incorrecto la pieza por el pivote o coloca una pieza que no corresponde en orden.

Colocación con problemas: el niño muestra tener dificultades para introducir correctamente la pieza por el pivote.

Autocorrección de errores: el niño corrige él mismo los errores de colocación que llevó a cabo previamente. 


\section{RESULTADOS}

El análisis de datos que sigue se divide en dos partes claramente diferenciadas. La primera de ellas, de naturaleza más clásica, se dirige a tratar de determinar la estructura factorial presente en nuestros datos, de cara a reducir en lo posible la gran variabilidad de información obtenida. A este respecto, hemos factorizado tanto las puntuaciones de intervención de los padres, cuanto las de desarrollo de los niños, de cara a manejar índices globales que nos permitan poner a prueba algunas hipótesis generales (existencia en los padres de distintos estilos de interacción, dependencia del nivel cultural, relación entre estilo de interacción de los padres y nivel de desarrollo del niño). La segunda parte del análisis de resultados nos parece particularmente indicada de cara a analizar procesos de interacción desde una perspectiva microgenética: se trata de un análisis secuencial de las interacciones adulto-niño, realizado con parte de los sujetos de la muestra, precisamente aquéllos que, sobre la base del análisis anterior, presentan estilos de interacción marcadamente distintos entre sí. Los índices que se manejan aquí son más finos y nos permiten, en consecuencia, poner a prueba algunas de nuestras hipótesis más específicas sobre el trabajo que adulto y niño realizan en la ZDP del niño.

\section{Análisis factorial y de regresión}

Como el número de piezas de la tarea con que jugaban los padres era mayor que el de la tarea de las madres y ello podía afectar al volumen de comportamientos desarrollados, antes de efectuar el análisis factorial compensamos las puntuaciones de padres y madres multiplicando las puntuaciones totales de los padres por la ratio resultante de dividir el número de piezas de la tarea de las madres entre el número de piezas de los padres $(12: 16=0,75)$.

De cara a la realización del análisis factorial, hemos seleccionado ocho variables criterio que nos parecían especialmente significativas tras análisis exploratorios previos. Entre estas variables faltan algunas que para nosotros eran inicialmente de especial interés, pero que los análisis exploratorios mostraron que se comportaban de acuerdo con una lógica distinta a la prevista. Así ha ocurrido, por ejemplo, con la verbalización de aprobaciones: probablemente porque los padres eran conscientes de que la tarea era muy difícil para los niños, todos ellos eran en general muy generosos con las aprobaciones, con independencia del comportamiento del niño; DeLoache y DeMendoza (1987), que han informado de un dato muy semejante, lo interpretan como un recurso para hacer la interacción más satisfactoria tanto para los adultos como para los niños. Algunas de las variables retenidas no son "variables puras", sino, por así decirlo, "variables secuenciales", como es el caso de "Aporte de material de entrada", que se codifica cuando la categoría "Aporte de material» aparece sin que el niño haya hecho esfuerzo previo alguno de cara a elección de la pieza que corresponda. Creemos que estas «categorías secuenciales» tienen un significado psicológico mucho más claro e informativo que las categorías puras; así, por ejemplo, no es lo mismo considerar "Desaprobación» sin más, que considerar «Desaprobación seguida de explicaciones», pues en uno y otro caso, el va- 
lor psicológico de la conducta de desaprobación es muy diferente. Las ocho categorias retenidas para el análisis factorial han sido: Instrucción verbal específica, Regla parcial de elección, Demora en la situación de elección, Demora tras una colocación con problemas, Desaprobación seguida de explicaciones, Aporte de material de entrada, Toma de posesión de la actividad, Ayuda física de entrada en la colocación de la pieza.

El análisis factorial de las puntuaciones de los padres se ha llevado a cabo siguiendo el procedimiento de la máxima probabilidad (Jöreskog, 1967; Jöreskog y Lawley, 1968), que trata de encontrar la solución factorial que mejor se ajuste a las correlaciones observadas entre las variables y que además basa el análisis en la varianza explicada por las variables en estudio. El análisis revela la existencia de dos factores que presentan una elevada correlación entre sí $(0,33$, lo que es un valor apreciable si se considera que lo que se relacionan son factores y no puntuaciones). Hemos retenido aquél que presenta unos pesos factoriales más elevados. En él, las categorías que pueden ser consideradas representativas de una interacción estimulante presentan en general pesos positivos elevados (Instrucción verbal específica, 0,97; Regla parcial de elección, 0,84; Demora en la elección, 0,93; Desaprobación seguida de explicaciones, 0,60 ), con la única excepción de la Demora tras una colocación con problemas, que adquiere un peso negativo bajo en este factor $(-0,19)$. Por otro lado, las categorías que pueden ser consideradas representativas de una interacción no estimulante, adquieren un peso negativo sobre el mismo factor, como es el caso de Aporte de material de entrada $(-0,52)$, Toma de posesión de la tarea $(-0,14)$ y Ayuda de entrada $(-0,14)$. El segundo factor presenta en general pesos factoriales bajos, excepto en la ya mencionada variable Demora tras una colocación con problemas $(0,98)$, que conceptualmente corresponde a los valores positivos del otro factor.

Parece, por tanto, claro que el análisis factorial ha desvelado la existencia de un único factor, que bien podría denominarse "factor estimulación del desarrollo", con unos valores positivos en los que se encuentran los comportamientos de los padres que implican reto y demanda (Instrucción verbal específica, Regla parcial de elección, Demoras tras el fracaso o la dificultad, Desaprobación seguida de explicaciones), y unos valores negativos que agrupan a variables que implican lo contrario: intervención directa por parte de los padres, sin retos ni demandas (Aporte de material de entrada, Ayuda de entrada, Toma de posesión de la tarea).

La matriz de correlaciones confirma la estructura interna del factor y su significación psicológica. Así, por ejemplo, la correlación entre Instrucción verbal específica y Regla parcial de elección es de 0,83; entre Regla parcial de elección y Demora en la elección, 0,77; entre Demora en la elección y Demora tras una colocación con problemas, 0,21. Las correlaciones son negativas en los casos en que se implican las variables ligadas con las interacciones menos estimulantes: Instrucción verbal específica y Apoyo de entrada -0,52; Demora en la elección y Ayuda de entrada, -0,15; Demora tras problemas de colocación y Toma de posesión de la tarea, $-0,30$.

Similar procedimiento se ha seguido en el caso de las puntuaciones de los niños en los diversos aspectos considerados: índice mental de Bayley, Número de raices diferentes, Total de palabras. En el «factor desarrollo» resultante tienen un peso elevado Total de palabras $(0,97)$ y Número de raí- 
ces diferentes $(0,96)$, seguidos de Indice Bayley $(0,51)$. La matriz de correlaciones muestra la estrecha asociación entre las dos puntuaciones referidas a lenguaje ( 0,93 entre Total de palabras y Número de raíces diferentes), así como una buena correlación entre las puntuaciones Bayley y las de lenguaje $(0,49$ en los dos casos).

Una vez determinados los dos factores «estimulación" y "desarrollo", es posible conocer el valor estimado del factor en cada uno de los sujetos: qué valor estimado tienen las puntaciones de cada uno de nuestros padres en el factor estimulación, y cuál tienen cada uno de nuestros niños en el factor desarrollo. Establecidas estas estimaciones por el método de la regresión, es posible proceder a calcular la recta de regresión entre ambas columnas de valores, utilizando como variable dependiente las estimaciones de desarrollo de los niños y como predictoras las estimaciones de estimulación de sus padres. Al hacerlo, obtenemos un valor de $r$ de 0,18, que es claramente bajo, pero significativamente distinto de $0(F=4,58, p<0,05)$. Así, pues, hay una correlación significativa, aunque baja, entre las puntuaciones de estimulación de los padres y las de desarrollo de los niños.

Naturalmente, cada niño tiene una sola estimación de desarrollo, pero dos estimaciones de estimulación referidas a su padre y su madre, respectivamente. Resulta, por tanto, razonable preguntarse si las estimaciones de desarrollo del niño se predicen mejor, peor o igual cuando se toma como variable independiente la estimación de estimulación de la madre, o cuando se toma la del padre. El procedimiento consiste simplemente en hacer dos rectas de regresión, una en la que se consideran las estimaciones de las madres en su factor y las de los niños en el suyo, y otra en la que se toman los valores estimados de los padres en su factor y los de los niños en el suyo. Al operar así, se obtuvo que, considerando aisladamente las puntuaciones de los padres, la correlación entre el factor estimulación y el factor desarrollo bajaba $(0,17)$ y el índice de regresión perdía toda significatividad $(F=1,95, p=0,16)$. Considerando sólo a las madres, la correlación entre ambos factores aumentaba $(r=0,23)$, pero el valor del índice de la regres sión descendía $(F=3,57)$ y, aunque perdía significatividad al $95 \%$, se mantenía muy cercano a ese límite $(p=0,06)$.

De acuerdo con una de nuestras hipótesis, hemos analizado en qué medida el carácter más o menos estimulante del comportamiento de los padres se relaciona con su nivel de estudios. El análisis de varianza 3 (niveles de estudios) $\times 2$ (sexo), tomando como variable dependiente los valores estimados de estimulación, muestra que, contrariamente a nuestras expectativas, no es el nivel educativo de los padres $(F=1,59, p>0,05)$, sino su sexo $(F=3,92, p<0,05)$ lo que se relaciona con la estimulación aportada a los niños durante la interacción aquí analizada. La interacción sexo-nivel de estudios no da tampoco lugar a valores significativos. El examen de las puntuaciones medias de los adultos de cada nivel de estudios muestra que la media de las puntuaciones en estimulación de los de nivel educativo bajo es negativa $(-0,18)$, que la media de los de nivel educativo alto es positiva $(0,20)$ y que la media de los sujetos de nivel de estudios medio está entre las anteriores $(0,01)$. No obstante, las desviaciones estándar intragrupo (sobre todo en los sujetos de niveles medio y alto) son lo suficientemente elevadas como para impedir que la relación alcance valores significativos.

En lo que a las diferencias hombres-mujeres concierne, es interesante 
hacer notar que el valor medio de las estimaciones de puntuación en el factor en el caso de las mujeres es de $-0,17$, mientras que en el caso de los padres es 0,17 , lo que induciría a pensar que las madres presentan niveles de estimulación más bajos que los hombres. Lo que ocurre en realidad es que en el caso de los hombres hay algunas puntuaciones de estimulación muy elevadas, lo que sube mucho la media de su grupo. Así lo muestra que la desviación tipo de las madres es 0,69 , mientras que en el caso de los hombres la desviación tipo correspondiente es 1,19 .

\section{Análisis secuencial}

De cara a completar la prueba de las hipótesis planteadas, se ha efectuado además un análisis más pormenorizado de las interacciones desarrolladas entre las madres que puntuaban más alto y aquéllas que puntuaban más bajo en el factor de estimulación con sus respectivos hijos. La elección sólo de madres está motivada por el hecho de que, tal y como se ha reseñado más arriba, parece ser su comportamiento, más que el de los padres, el que mejor predice el nivel de desarrollo de los niños. Se estudiaron las 8 madres que puntuaban más bajo en el factor y las 7 que puntuaban más alto. La elección de un número desigual de madres se debió al valor mínimo de puntuación en el factor elegido como límite en cada caso. De todas formas, el hecho de tener un número desigual no dificulta la comparación de los datos, al trabajar fundamentalmente con medias, probabilidades y puntuaciones tipificadas.

Como ocurría en el caso del análisis anterior, algunas de las categorías de codificación no han sido tomadas en consideración, en este caso por su frecuencia de aparición o muy baja o nula. Tal es el caso de categorías como Regla de la tarea, Petición al niño de autocorrección y Autocorrección de errores. Con Expectativas positivas y Aprobación vuelve a ocurrir lo mismo que en el análisis anterior: en general, todas las madres las manejan con una generosidad no dependiente de la ejecución del niño en la tarea.

El análisis secuencial nos permite tomar algunos momentos críticos de la ejecución de la tarea (por ejemplo, un error del niño, o la intervención del padre consistente en darle al niño la pieza que tiene que colocar) y evaluar qué probabilidad existe de que el comportamiento de que se trate aparezca asociado a otro comportamiento que se considere de interés: por ejemplo, qué probabilidad existe de que a un error del niño le siga una demora o una corrección por parte del padre, o qué probabilidad hay de que un padre dé al niño una pieza directamente, sin que haya habido por parte del niño esfuerzo alguno de elegir la correcta, o por parte del padre algún intento de facilitarle la elección en vez de resolvérsela directamente. Este tipo de análisis nos permite valorar cuál es el planteamiento que los padres hacen al niño de la tarea y cómo van variando sus exigencias y sus apoyos en función đel comportamiento que observan en él. Para estos análisis hemos utilizado la versión 4 del programa ELAG (Bakeman, 1986).

Antes de entrar en los datos aportados por el análisis secuencial, puede ser útil hacerse una idea previa de lo que ocurre en general en el conjunto de las interacciones, y para ello hemos efectuado un contraste de medias en el que consideramos las diferentes categorías que hemos utilizado en el 


\section{2}

TABLA II

Contraste de medias entre los dos grupos de madres para las categorias relacionadas con los niveles de intervención en la tarea por parte de los padres $y$ con las realizaciones de los niños

\begin{tabular}{|c|c|c|c|c|c|c|c|}
\hline & \multicolumn{3}{|c|}{$\begin{array}{c}\text { Madres } \\
\text { estimulantes }\end{array}$} & \multicolumn{3}{|c|}{$\begin{array}{l}\text { Madres poco } \\
\text { estimulantes }\end{array}$} & \multirow{2}{*}{$\mathbf{t}$} \\
\hline & $\Sigma$ & $\overline{\mathbf{X}}$ & $\sigma$ & $\Sigma$ & $\overline{\mathbf{X}}$ & $\sigma$ & \\
\hline $\begin{array}{l}\text { Instrucción verbal inespecí- } \\
\text { fica }\end{array}$ & 5 & 0,71 & 0,88 & - & 一 & - & \\
\hline $\begin{array}{l}\text { Instrucción verbal específi- } \\
\mathrm{ca}\end{array}$ & 47 & 6,71 & 1,03 & - & 一 & - & \\
\hline Elección correcta & 38 & 5,42 & 2,19 & 10 & 1,25 & 1,71 & 3,61 \\
\hline Elección incorrecta & 51 & 7,28 & 2,18 & 20 & 2,50 & 3,77 & 2,64 \\
\hline Desaprobaciones & 80 & 11.42 & 3,15 & 21 & 2,62 & 2,11 & 6,26 \\
\hline Aporte de material & 35 & 5 & 2,67 & 75 & 9,37 & 3,73 & 2,79 \\
\hline $\begin{array}{l}\text { Orientación correcta de la } \\
\text { pieza }\end{array}$ & 44 & 6,28 & 5,22 & 29 & 3,62 & 3,63 & 1,02 \\
\hline $\begin{array}{l}\text { Ayuda física en la coloca- } \\
\text { ción }\end{array}$ & 16 & 2,28 & 0,69 & 13 & 1,85 & 1,55 & 1,07 \\
\hline $\begin{array}{l}\text { Toma de posesión de la ac- } \\
\text { tividad }\end{array}$ & 11 & 1,57 & 1,59 & 28 & 3,50 & 4,47 & 1,03 \\
\hline Colocación correcta & 60 & 8,57 & 2,06 & 52 & 6,50 & 4,66 & 0,79 \\
\hline Colocación con problemas & 62 & 8,85 & 3,75 & 37 & 4,62 & 3,27 & 2,02 \\
\hline Colocación incorrecta & 26 & 3,71 & 1,74 & 23 & 2,87 & 3,91 & 0,48 \\
\hline
\end{tabular}

: Significativo para $p=0,05$.

$\because$ Significativo para $p=0,01$.

Significativo para $p=0,001$

análisis secuencial (Tabla II). Merece la pena destacarse el hecho de que las instrucciones verbales (ya sea las pocas inespecificas que se observan, ya sea el volumen mayor de específicas), aparezcan sólo en el grupo de madres estimulantes. Es también digno de resaltarse el hecho de que sea significativa la diferencia en la conducta de Aporte de material (dar al niño la pieza que hay que colocar), en este caso con una mayor incidencia en las madres menos estimulantes. Resulta de interés notar la diferencia significativa que se produce en la verbalización de Desaprobaciones, conducta en la que las madres más estimulantes superan significativamente a las menos estimulantes. Las diferencias son también significativas entre un grupo y 
otro en las conductas de elección por parte de los niños (tanto en el caso de las elecciones correctas como de las incorrectas, los hijos de madres estimulantes superan significativamente a los de las madres menos estimulantes), así como en la Colocación con problemas, de nuevo a favor de los hijos de madres más estimulantes (en el resto de las conductas de colocación, los hijos de madres estimulantes tienen medias superiores a los de madres menos estimulantes, pero la amplitud de las desviaciones tipo hace que no alcancen valores significativos). Algo semejante ocurre en las conductas de ayudar al niño orientándole correctamente la pieza o facilitándose su encaje en el pivote, en las que las madres más estimulantes tienen medias superiores a las menos estimulantes, pero en las que las desviaciones tipo son altas. Lo contrario ocurre en la conducta materna de Toma de posesión de la tarea, en la que las madres menos estimulantes superan a las estimulantes en la media, sin que las diferencias adquieran valores significativos. Las diferencias entre los grupos tampoco son significativas para el resto de las conductas de colocación, aunque la media de las colocaciones correctas es claramente superior en el caso de los hijos de madres estimulantes.

Otro contraste interesante entre las madres que puntúan alto en el factor estimulación y las que puntúan bajo, concierne a un aspecto muy relacionado con la definición de la situación que van haciendo al niño a lo largo de la interacción: ¿hasta qué punto las madres plantean la tarea como una actividad gobernada por ciertas reglas que rigen la elección y la colocación de todas las piezas? $\mathrm{El}$ análisis del volumen de reglas que las madres verbalizan (Reglas generales de la tarea, Regla parcial de elección, Regla parcial de colocación) nos puede permitir saber en qué medida las madres intentan aportar al niño a lo largo de la tarea un instrumento conceptual (las reglas) que es útil para el conjunto de la tarea (y no sólo para una pieza determinada), o más bien actúan como si no hubiera una lógica común y repetida de unas piezas a otras. Para responder a estos interrogantes, hemos procedido a un contraste de medias entre las madres que puntúan alto y bajo en estimulación considerando las reglas parciales de elección, las reglas parciales de colocación y el volumen total de reglas parciales, sean de elección o colocación. Las reglas generales de la tarea no han aparecido en las madres aquí analizadas. Como muestra la Tabla III, aunque hay una sustancial diferencia en el volumen de reglas parciales de elección entre uno y otro grupo, esta diferencia no es significativa, probablemente debido de nuevo a la desviación tipo excesivamente alta de los datos. Tampoco es significativa la diferencia entre las medias de reglas parciales de colocación. Sí es significativa, sin embargo, la diferencia entre los dos grupos cuando se toma el volumen total de reglas parciales: las madres más estimulantes presentan un volumen de reglas parciales significativamente más elevado que el de las madres menos estimulantes.

Por lo que al análisis secuencial se refiere, hemos interrogado a nuestros datos en torno a unos cuantos hitos de especial significación en la tarea: ¿qué ocurre tras una instrucción verbal del tipo "coge ahora la más grande de las amarillas»?; ¿qué conducta siguen las madres tras un error del niño, ya sea la elección de una pieza equivocada, ya la colocación total o parcialmente incorrecta de una pieza?; ¿cuál es la situación previa al momento en que la madre decide resolver el problema al niño, ya sea dándole la pieza que se ha de colocar, ya sea colocándola por él? 


\section{4}

TABLA III

Contraste de medias entre los dos grupos de madres, referido a la utilización de reglas parciales

\begin{tabular}{|l|ccc|cccc|c|}
\hline & \multicolumn{3}{|c|}{$\begin{array}{c}\text { Madres } \\
\text { estimulantes }\end{array}$} & \multicolumn{3}{c|}{$\begin{array}{c}\text { Madres poco } \\
\text { estimulantes }\end{array}$} & \multirow{2}{*}{$t$} \\
\cline { 2 - 7 } & $\Sigma$ & $\overline{\mathbf{X}}$ & $\mathbf{0}$ & $\Sigma$ & $\overline{\mathrm{X}}$ & $\sigma$ & \\
\hline Regla parcial de elección & 57 & 8,14 & 4,70 & 4 & 0,5 & 1 & 1,18 \\
\hline Regla parcial de colocación & 60 & 8,57 & 3,06 & 45 & 5,62 & 5,12 & 0,26 \\
\hline Total de reglas parciales & 117 & 16,71 & 5,92 & 49 & 6,12 & 5,06 & 3,24 \\
\hline
\end{tabular}

$\approx *$ Significativo para $p=0,001$.

Lo primero que nos preguntamos es qué ocurre tras una Instrucción verbal por parte de la madre. Caso de que la madre utilice tales instrucciones, puede ocurrir que el niño elija correctamente la pieza, o que se equivoque al elegirla, en cuyo caso la madre puede volver a intentarlo con una nueva Instrucción verbal (lo que implica mantener la exigencia y demorar el apoyo), o puede dar al niño la pieza que hay que colocar. Como muestra la Tabla III, las instrucciones verbales sólo han aparecido en el grupo de las madres con puntuaciones estimadas altas en el factor estimulación. En ellas, aparece un cierto número de instrucciones verbales consecuentes a un fracaso del niño, aunque los valores $z$ no son significativos.

Nuestro segundo interrogante es qué conducta siguen las madres tras un error por parte del niño. Se trata de saber si en tales casos (Elección incorrecta, Colocación total o parcialmente incorrecta) las madres reaccionan con una intervención que sigue retando al niño y demandando su esfuerzo (Instrucción verbal en el caso de elección; «apoyo estimulante» en el caso de colocación, lo que incluye reglas parciales de colocación, verbalización de expectativas positivas y demoras), o más bien actúan apoyando directamente al niño, sin demoras (elegir la pieza por el niño, orientarla correctamente, ayudarle físicamente en la colocación o simplemente colocarla ella). Los datos de la Tabla III muestran que en el caso de los errores en la elección, la $\mathrm{X}^{2}$ que compara las proporciones obtenida en los dos grupos es significativa. Lo mismo ocurre tras una colocación incorrecta: los valores de $z$ no son significativos, pero sí lo es el contraste entre los dos tipos de madres, que da lugar a una $\mathrm{X}^{2}$ significativa.

Finalmente, nuestro tercer interrogante se refiere a un aspecto en parte relacionado con el anterior: cuál es la situación previa al momento en que la madre decide resolver el problema al niño, ya sea dándole la pieza que se ha de colocar, ya sea colocándola por él. Tal intervención materna puede ocurrir tras un error por parte del niño, ya sea en elección o en colocación, o antes de haber dado a éste oportunidad de equivocarse en cualquiera de las dos subtareas, lo que supone claramente una menor estimulación, al no dar al niño oportunidad para intentar resolver el problema, con las interacciones que pueden darse alrededor de tales intentos. Como se observa en la Tabla IV, hay una desigual distribución de patrones según se tra- 


\section{5}

TABLA IV

Datos procedentes del análisis secuencial

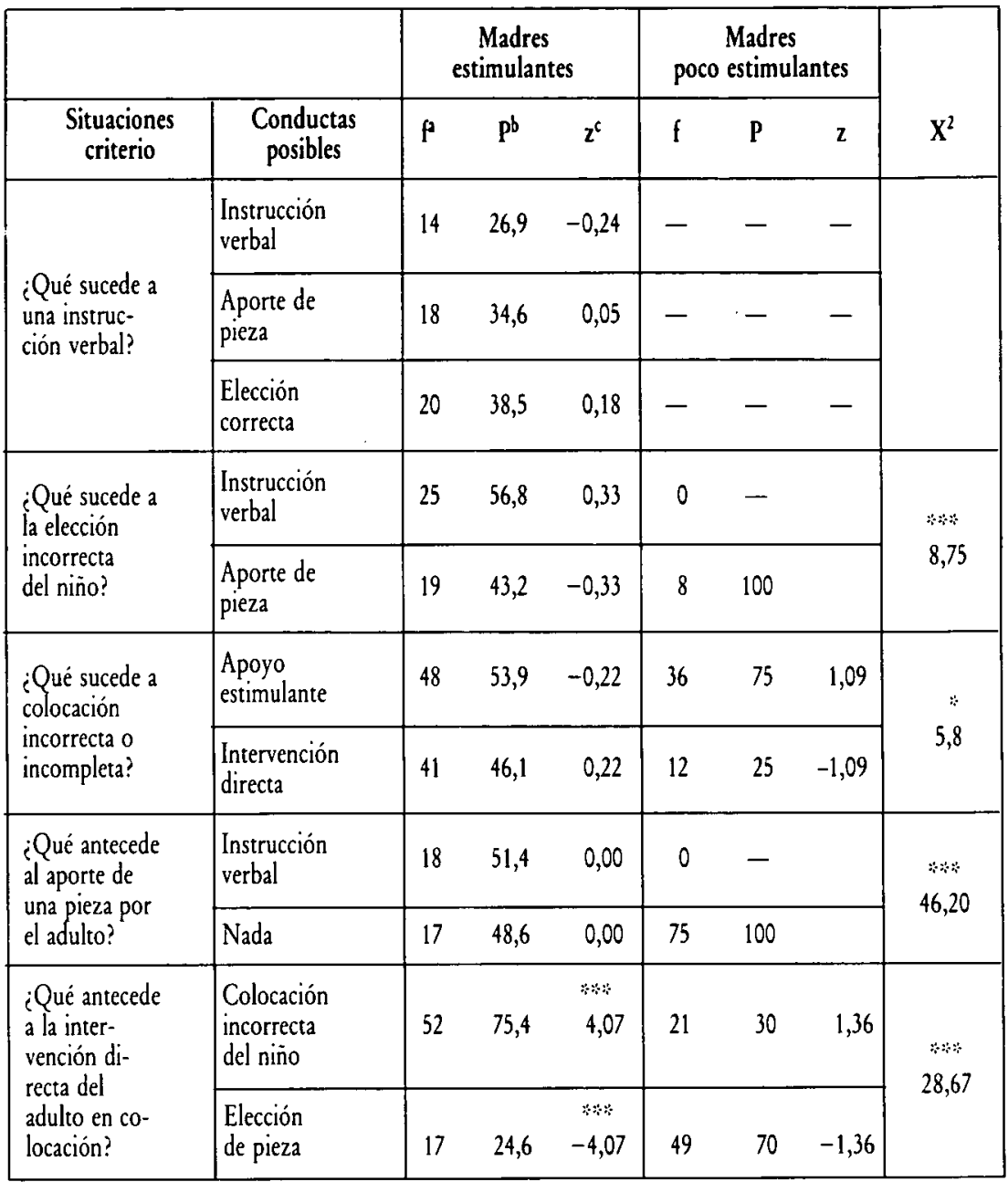

$\mathrm{f}^{2} \quad=$ Frecuencia de aparición.

$\mathrm{P}^{\mathrm{b}} \quad=$ Probabilidad de asociación con la conducta criterio.

$z^{\mathrm{c}} \quad=$ El índice $z$ evalúa en este caso la significatividad de un valor de probabilidad transicional, es decir, la probabilidad de que, dado un determinado comportamiento, le anteceda o le suceda otro. Para ello toma en consideración no sólo los distintos patrones secuenciales que pueden aparecer, sino también las frecuencias absolutas de aparición de los distintos comportamientos.

\footnotetext{
* Significativo para $p=0,05$.

*: Significativo para $p=0,01$.

Significativo para $p=0,001$.
}

te de elección o de colocación. En el caso de la intervención directa en colocación, las madres estimulantes la introducen con mucha mayor probabilidad tras un error del niño que antes de que el error se haya producido; en el caso de las madres menos estimulantes, la intervención de la madre se produce con mayor probabilidad antes de que el niño haya podido equivocarse. El contraste $\mathrm{X}^{2}$ entre los dos grupos muestra que las diferencias son significativas. 
En el caso de la intervención directa de la madre (aportar la pieza) en la subtarea de elección, los valores de $z$ obtenidos en las madres estimulantes no son significativos, en contraste con lo que ocurre con las madres menos estimulantes, en las que el $100 \%$ de las intervenciones se producen sin haber dado al niño ocasión de equivocarse. Los contrastes de $\mathrm{X}^{2}$, que comparan las proporciones obtenidas en las dos muestras, son de nuevo significativos.

\section{DISCUSION}

Los datos procedentes de los dos tipos de análisis efectuados (factorial y secuencial), muestran claramente que los adultos difieren unos de otros en su forma de interactuar educativamente con los niños, y lo hacen a lo largo de un continuum que va desde los niveles más elevados de estimulación hasta los más bajos. La posición de un sujeto determinado en uno u otro lugar del continuum, parece estar relacionada con determinadas variables. Mientras que nuestra hipótesis preveía que el nivel educativo de los padres guardaría una estrecha relación con su posición en uno u otro punto del continuum, tal y como se ha encontrado en múltiples investigaciones (por ejemplo, Laosa, 1983), los datos que hemos presentado muestran que es el sexo y no el nivel de estudios de los padres lo que más estrechamente se relaciona con la calidad de la estimulación que aportan a sus hijos. Nuestros datos muestran unas puntuaciones medias de estimulación más altas en los padres que en las madres, lo que probablemente se deba a las elevadas puntuaciones alcanzadas por algunos padres. Pero el dato más relevante a este respecto es que son las puntuaciones de las madres ( $y$ no las de los padres) las que parecen relacionarse más claramente con las puntuaciones de desarrollo de los niños. Este hecho merece, a nuestro juicio, algunas consideraciones.

La "especialización» de las madres en la educación de los hijos es uno de los usos ampliamente extendidos en nuestra cultura. Ellas suelen pasar con los bebés la mayor parte del tiempo, interactúan con ellos asiduamente, etc. Mucho más frecuentemente que los padres, las madres están implicadas con sus hijos en interacciones de carácter educativo. Los padres, más frecuentemente, interactúan con sus hijos más en términos de juego. Tal vez una forma de entender que el nivel de estimulación en la tarea de construcción que hemos analizado se relacione tan fuertemente con el sexo, proceda del hecho de que se trata de una tarea muy estructurada cuya realización estimulante requiere una actitud claramente educativa, y no meramente lúdica. Requiere además un fino ajuste entre las capacidades que el niño tiene y las exigencias y apoyos que se le van planteando, lo que implica no sólo tener una intencionalidad educativa, sino también una cierta práctica en este tipo de interacciones y un buen conocimiento del niño y sus posibilidades. Quizá eso ocurra mucho más en el caso de las madres que de los padres (pudiera ser que las puntuaciones llamativamente altas que unos pocos padres presentan en estimulación reflejen un menor conocimiento del niño y sus posibilidades). Cabe formular la hipótesis de que con tareas menos estructuradas que la que hemos utilizado para nuestros análisis, las diferencias ligadas al nivel educativo se hagan más patentes. Esta 
hipótesis será puesta a prueba en el análisis de las interacciones que los mismos sujetos que han sido analizados aquí han mantenido en situaciones bastante diferentes a la ahora analizada (lectura de un cuento a base de imágenes y juego con materiales caseros de desecho). Como complemento a esta hipótesis, se puede pensar que los comportamientos observados son una consecuencia no sólo de las características de la tarea, sino de la interacción entre tales características y la edad de los niños: puede que los padres (varones) se inclinen menos aún a adoptar un estilo interactivo típicamente educativo en el caso de niños pequeños que en el de niños mayores. Esta hipótesis se pondrá a prueba cuando estos mismos sujetos sean evaluados con tareas semejantes cuando los niños tengan 5 años. Sea como quiera, las diferencias aquí encontradas entre hombres y mujeres han sido halladas también por otros investigadores, que, como en el caso de Lamb, Frodi, Frodj y Hwang (1982), han sugerido además que las diferencias habituales hombres-mujeres en la interacción educativa con los bebés pueden acentuarse más aún cuando unos y otras se sienten observados.

La otra hipótesis general que sosteníamos (el lugar que los padres ocupan en el continuum del factor estimulación correlaciona con el que ocupan los niños en el del factor desarrollo) ha encontrado una confirmación parcial. La correlación ha resultado ser, en conjunto, significativa, pero baja. Cuando se toma en consideración sólo a las madres, el valor de la correlación aumenta, mientras que cuando se considera sólo a lo padres, la correlación baja. Este hecho está, a nuestro entender, relacionado con el que acabamos de comentar en el párrafo anterior. Son de hecho las madres quienes más se implican en la interacción educativa cotidiana con sus hijos, y, en coherencia con el principio del origen interpersonal de los procesos intrapersonales, es predecible entonces que las puntuaciones de desarrollo estén relacionadas con las de la estimulación cotidiana. Ello hace que, incluso si algunos padres tienen una notable capacidad para estimular a los niños en interacciones educativas, al ser esas interacciones escasas o infrecuentes, eso no redunde en la correspondiente traducción intrapersonal de esas interacciones.

Cabe, no obstante, preguntarse por qué razón la correlación encontrada es baja $(0,23)$. Varias interpretaciones son posibles. Una de ellas tiene que ver con los índices utilizados, que, hasta cierto punto, tal vez sean poco coherentes entre sí. El factor desarrollo de los niños ha resultado estar muy cargado de contenido verbal (las dos variables con más peso en él son Total de palabras y Total de raices diferentes, con una elevada correlación entre sí), mientras que el factor estimulación en los padres no está concebido para valorar específicamente contenidos de ese tipo. Nuestra previsión es que cuando el factor desarrollo se ponga en relación con la estimulación aportada por los padres en la tarea de «lectura» de un cuento, el valor de la correlación aumentará, pues los contenidos de los dos índices serán más coherentes.

Una interpretación no incompatible con la anterior se orienta en una dirección muy diferente. Como algunos autores han sostenido, el desarrollo de los bebés está fuertemente "canalizado" (ver, por ejemplo, McCall, 1981), de tal modo que incluso en circunstancias ambientales muy diversas, los niños menores de 18-24 meses, tienden a presentar perfiles evolutivos similares y muy dependientes de la maduración de estructuras de base. Lo 
anterior no significa que los bebés sean insensibles a la estimulación, o que su desarrollo esté previsto en los genes. Significa que los efectos de la estimulación se irán haciendo más evidentes a medida que el perfil evolutivo esté menos canalizado. Si esta hipótesis es cierta, cuando nuestros sujetos sean estudiados de nuevo al cumplir los niños 5 años, la correlación estimulación-desarrollo deberá ser más elevada. Ello además no impide que esa correlación sea especialmente más elevada cuando entonces se pongan en relación categorías de estimulación y de desarrollo en el mismo ámbito (por ejemplo, el lenguaje), de acuerdo con nuestra hipótesis anterior.

Son los datos del análisis secuencial los que sin duda encajan mejor con nuestras hipótesis. Existe una notable diferencia en la forma en que los dos grupos de madres considerados definen la situación para los niños: las unas como una tarea gobernada por reglas, las otras como una tarea en cuyo planteamiento no se destaca lo que en ella puede haber de "conceptual" ( $\mathrm{Ta}$ blas II y III). Naturalmente, definir la situación en términos de reglas es hacerla más difícil, lo que implica, para expresarlo en los términos de Wood (1980), poner el andamio más alto. De manera interesante, sin embargo, el andamio no se sube demasiado alto, como lo demuestra el hecho de que las conductas de más elevado nivel de demanda conceptual o no hayan aparecido (Regla de la tarea), o hayan aparecido escasamente (Instrucciones verbales inespecíficas), lo que debe entenderse como una muestra de sensibilidad respecto a lo que podríamos denominar «el techo» de las posibilidades que los niños tienen.

Tal vez estamos ante dos formas muy diferentes de concebir la tarea por parte de las madres: para unas, las más estimulantes, estamos ante una tarea con un fuerte componente conceptual, donde las reglas son importantes; para otras, estamos ante una tarea con un fuerte componente sensoriomotor, en la que las reglas son menos importantes. Si esta hipótesis fuera correcta, la distinta forma de comportarse deriva en buena parte de distintas formas de entender la tarea, diferencia que, de existir, sería en sí misma muy reveladora.

Esa presencia de un mayor nivel de exigencias en unas madres que en otras, se observa también en el planteamiento de instrucciones verbales por parte de las madres más estimulantes, que, para decirlo en términos de Wertsch (1985), desafian de esa forma más a sus hijos (Tablas II y IV). En contraste, las madres menos estimulantes presentan un nivel mucho más elevado de interacciones en las que son ellas quienes eligen la pieza y se la dan al niño. Es muy destacable que en el $100 \%$ de los casos, la conducta de dar al niño la pieza sea una conducta "de entrada" en el caso de las madres menos estimulantes, mientras que en el $50 \%$ de los casos de las madres más estimulantes, antes de dar al niño la pieza correspondiente, ha habido una instrucción verbal (Tabla IV). Los datos referidos a qué antecede a la intervención directa de la madre en la subtarea de colocación confirman el mismo hecho: en el $75 \%$ de los casos, las madres estimulantes intevienen en la colocación de la pieza sólo después de que el niño lo haya intentado y haya fracasado; en el $70 \%$ de los casos, las madres poco estimulantes colocan ellas la pieza en general tras haberla seleccionado ellas mismas (en unos pocos casos, colocan ellas la pieza una vez que el niño la ha elegido al azar).

Pero las madres más estimulantes no se limitan a retar más a los niños: 
mantienen más sus desafíos. Cuando el niño elige incorrectamente una pie$\mathrm{za}$, si su madre es poco estimulante, en el $100 \%$ de los casos recibe la pieza directamente; si, por el contrario, su madre es más estimulante, en el $57 \%$ de los casos, el niño recibe una nueva instrucción verbal. Este estado de cosas se invierte, curiosamente y en contra de nuestras hipótesis, en la subtarea de colocación, donde si el niño fracasa es más probable que reciba un apoyo estimulante por parte de las madres menos estimulantes que por parte de las que lo son más. Ello tal vez se deba a que las madres actúan con una lógica distinta en colocación que en elección, lo cual puede relacionarse con las dos formas distintas de concebir la tarea por parte de las madres a que nos hemos referido más arriba: las que destacan más el lado conceptual de la tarea, suben sus exigencias en elección y las bajan en colocación, mientras que las que destacan el lado sensoriomotor de la tarea, son más estimulantes en colocación que en elección.

De todas formas, lo que ocurre con las desaprobaciones es también una buena prueba de la forma en que las madres más estimulantes no sólo plantean a los niños exigencias más elevadas, sino que además las mantienen más consistentemente: la notable diferencia que hay en desaprobaciones a favor de las madres más estimulantes, señala que se da al niño un feedback correctivo cuando comete errores (lo que ocurre con frecuencia, pues las exigencias son mayores), feedback que no se limita a señalar el error, sino que, como hemos visto, saca de él provecho para explicar reglas, dar nuevas instrucciones verbales, etc.

A la luz de estas consideraciones se entienden bien algunos de los datos de la Tabla II. Los hijos de madres más estimulantes se enfrentan con mucha más frecuencia a la tarea de elección que los hijos de madres menos estimulantes, pues éstas dan al niño la pieza directamente con mucha mayor frecuencia. Eso explica que los niños de madres estimulantes cometan muchos más errores en elección. Pero como estas madres no se limitan a permitir a los niños enfrentarse con el problema, sino que además les dan más medios para hacer frente a la situación, sus hijos superan claramente a los de las madres menos estimulantes también en lo que a elecciones correctas se refiere.

Una tendencia semejante se observa en la subtarea de colocación, aunque en este caso las diferencias no son tan claras. Los hijos de madres estimulantes se sitúan por encima de los otros en el número de colocaciones correctas (aunque en este caso las diferencias no son significativas y a pesar del mayor apoyo que, como vimos, los hijos de madres menos estimulantes reciben en este aspecto) y en el número de colocaciones con problemas (en este caso con valores significativamente diferentes). Los dos grupos no difieren en colocaciones incorrectas.

Aunque en este caso las diferencias no alcancen valores significativos como consecuencia de las desviaciones tipo elevadas, la tendencia de los datos es coherente con lo anterior cuando se consideran las variables Orientación correcta de la pieza y Toma de posesión de la actividad: la media de las madres estimulantes es más alta respecto a la primera (que implica apoyar la acción del niño, no suplantarla), mientras que la de las madres menos estimulantes es más elevada en la segunda (que implica, en efecto, realizar la acción por el niño).

En conjunto, pues, el análisis microgenético de lo que ocurre en las in- 
teracciones madre-hijo en la ZDP y alrededor de una tarea de construcción, muestra que en el curso de las interacciones se definen contextos de interacción, se establece una relación intersubjetiva a uno u otro nivel, se plantean exigencias y se aportan apoyos y se es más o menos consistente en el comportamiento que se desarrolla. Esta consistencia se da en unos casos en dirección estimulante, $\mathrm{y}$ en otros en dirección menos estimulante. El niño adquiere competencia en mayor o menor medida en función del contenido de estas interacciones. Es la calidad de las interacciones la que decide qué se construye en la ZDP del niño.

Si nuestras hipótesis generales se han visto no confirmadas (relación del nivel de estudios con el comportamiento educativo), o se han visto sólo débilmente corroboradas por los datos (relación estimulación-desarrollo), ello puede deberse a las peculiaridades de la tarea utilizada (muy estructurada y demandando una clara intencionalidad educativa), de los índices usados (factor desarrollo muy cargado de contenidos verbales en comparación con factor estimulación), al hecho de que el desarrollo de los bebés esté fuertemente canalizado, o a una combinación de estas diversas razones. Los anälisis de las otras tareas en que los mismos padres han interactuado con sus hijos, y el seguimiento longitudinal de nuestra muestra nos dirán si, a pesar de los datos aquí obtenidos, nuestras hipótesis son correctas.

\section{Notas}

1 En las traducciones habituales se suele encontrar la expresión «la instrucción crea la ZDP del niño", pero tanto Rogoff y Wertsch $(1984$, p. 3), como Valsiner $(1988$, p. 144) enfatizan que el término original ruso obuchenie implica tanto a quien enseña como a quien aprende, recomendando que la traducción se exprese en estos términos: «El proceso de enseñanza/aprendizaje crea la ZDP del niño".

\section{Referencias}

Bakeman, R. (1986). ELAG. Versión 4. Atlanta, Ge.: Georgia State University.

BROWN, R. (1973). A first language. The early stages. Cambridge, Ma.: Harvard University Press.

Deloache, J. S. y DeMendozA, O. A. P. (1987). Joint picturebook interactions of mothers and 1-year-old children. British Jounal of Developmental Psychology, 5, 11-123.

JÖRESKOG, K. G. (1967). Some contributions to maximum likelihood factor analysis. Psychometrika, 32, 443-484.

JÖESKOG, K. G. y LAWLEY, D. N. (1968). New methods in maximum likelihood factor analysis. British Journal of Mathematical and Statistical Psychology, 21, 85-96.

LAmb, M. E., Frodi, A. M., FrodJ, M. y HwANG, C. P. (1982). Characteristics of maternal and paternal behavior in traditional and nontraditional Swedish families. International Journal of Behavioral Development, $5,131-141$.

LAOSA, L. M. (1982). Families as facilitators of children's intellectual development at 3 years of age. En L. M. Laosa e I. E. Sigel (eds.), Families as learning environments for children. New York: Plenum.

MCCALL, R. B. (1981). Nature-nurture and the two realms of development: a proposed integration with respect to mental development, Child Development, 52, 1-12.

MCLANE, J. B. (1987). Interaction, context, and the Zone of Proximal Development. En M. Hickman (ed.), Social and functional approaches to language and thought. Nueva York: Academic Press.

Miller, J. F. y Chapman, R. S. (1985). Systematic Analysis of Language Transcripts (SALT). Language Analysis Laboratory, University of Wisconsin.

ROGOFF, B. y GARDNER, W. (1984). Adult guidance of cognitive development. En B. Rogoff 
y J. Lave (eds.), Everyday cognition: its development in social context. Cambridge, Mass.: Harvard University Press.

RogofF, B., MALKIN, C. y GILBRIDE, K. (1984). Interaction with babies as guidance in development. En B. Rogoff y J. V. Wertsch (eds.), Children's learning in the "Zone of Proximal Development». New Directions for Child Development, n. ${ }^{\circ}$. San Francisco, Cal.: Jossey-Bass.

ROGOFF, B. y WeRTSCH, J. V. (1984). Editors' note. En B. Rogoff y J. V. Wertsch (eds.), Children's learning in the "Zone of Proximal Development.. New Directions for Child Development, n. ${ }^{\circ}$ 23. San Francisco, Cal.: Jossey-Bass.

ROMMETVEIT, R. (1985). Language acquisition as increasing linguistic structuring of experience and symbolic behavior control. En J. V. Wertsch (ed.), Culture, communication and cognition: Vygotskyan perspectives. Cambridge, Mass.: Cambridge University Press.

VAlsiner, J. (1988). Developmental Psychology in the Soviet Union. Brighton, Sussex: Harvester Press.

VILA, I. (1984). La competencia comunicativa en los dos primeros años de vida. Tesis doctoral no publicada. Universidad de Barcelona.

VYGOTSKY, L. S. (1978). Mind in society. The development of higher psychological processes. Cambridge, Mass.: Harvard University Press (Traducción castellana, El desarrollo de los procesos psicológicos superiores. Barcelona: Crítica, 1979).

WeRTSCH, J. V. (1984). The Zone of Proximal Development: some conceptual issues. En B. Rogoff y J. V. Wertsch (eds.), Children's learning in the "Zone of Proximal Development.. New Directions for Child Development, n. ${ }^{\circ} 23$, San Francisco, Cal.: Jossey-Bass.

WERTSCH, J. V. (1985). Vygotsky and the social formation of mind. Cambridge, Mass.: Harvard University Press (Traducción castellana, Vygotsky y la formación social de la mente. Barcelona: Paidós, 1988).

WERTSCH, J. V. (1987). «Problem solving in social interaction: a microgenetic analysis. En M. Hickman (ed.), Social and functional approaches to language and thought. Nueva York: Academic Press.

WERTSCH, J. V., MiNICK, N. y ARNS, F. J. (1984). The creation of context in joint problemsolving. En B. Rogoff y J. Lave (eds.), Everyday cognition: its development in social context. Cambridge, Mass.: Harvard University Press.

WOOD, D. (1980). Teaching the young child: some relationships between social interaction, language and thought. En D. Olson (ed.), The social foundations of language and thought. Nueva York: W. W. Norton.

WOOD, D., BRUNER, J. S. y ROSS, G. (1976). The role of tutoring in problem solving. Journal of Child Psychology and Psychiatry, 17, 89-99.

WOOD, D. y MIDDLETON, D. (1975). A study of assisted problem-solving. British Journal of Psychology, 66, 181-191.

\section{Extended summary}

Using a construction task beyond the children's current level of competence, adult-child interactions have been studied in a sample of 68 children (20-24 months old) and their parents. Two different data analyses have been carried out. First, a factor analysis has shown the existence of a «stimulation factor" in parents behaviors. In this factor, behaviors such as using linguistic demands, employing rules that explain the logic of the task, delaying a direct intervention from the adult, have positive weights. Behaviors such as direct intervention before any problem arises for the child and taking over children's activity, have negative weights in the factor. Children's scores of development in different domains (Bayley mental scale, language) have also been factorized, resulting in a developmental index where the higher weights correspond to the linguistic contents. Regression analysis carried out shows that there is a significant, but low, correlation between parents' stimulation factor and children's development score. The value of the correlation increases when only mothers' scores are taken into account, decreasing notably when only fathers' scores are considered. Parents' level of stimulation is related to sex, but not to level of academic attainment. 
This data is seen as a consequence of task characteristics, children's age, and the different patterns existing between mothers and fathers in their relationship with young children.

As a second step, and by means of a sequential analysis, the microgenetic process of interaction has been studied in two subsamples of highly and poorly stimulating mothers. Data shows that these groups differ from each other in the way they define the task and negotiate it with the child in the course of the interaction. More stimulating mothers focus the task in a more conceptual fashion, making the rules governing the task explicit to the child, whereas less stimulating mothers don't convey rules in their interaction. In the course of the interaction, more stimulating mothers challenge the child more, maintain their demands more and avoid solving the task for the child. As a logical outcome, children who have received more challenges, but also more conceptual support, and who have been less supplanted by their mothers, show higher levels of performance than those who have received less demands and more direct supports.

Taken as a whole, data in this research are compatible with a Vygotskyan interpretation, emphasizing the relevance of interactive processes and highlighting the construction of development that takes place where interactions are developed within children's Zone of Proximal Development, and are regulated by an interactive logic where certain basic rules are observed. 